\title{
Reconstituted high-density lipoproteins promote wound repair and blood flow recovery in response to ischemia in aged mice
}

Tania Tsatralis ${ }^{1}$, Anisyah Ridiandries ${ }^{1,2}$, Stacy Robertson ${ }^{1,2}$, Laura Z. Vanags ${ }^{1,2}$, Yuen Ting Lam ${ }^{1,2}$, Joanne T. M. Tan ${ }^{1,2}$, Martin K. C. Ng ${ }^{1,2,3}$ and Christina A. Bursill ${ }^{1,2^{*}}$

\begin{abstract}
Background: The average population age is increasing and the incidence of age-related vascular complications is rising in parallel. Impaired wound healing and disordered ischemia-mediated angiogenesis are key contributors to age-impaired vascular complications that can lead to amputation. High-density lipoproteins (HDL) have vasculo-protective properties and augment ischemia-driven angiogenesis in young animals. We aimed to determine the effect of reconstituted $\mathrm{HDL}$ ( $\mathrm{rHDL}$ ) on aged mice in a murine wound healing model and the hindlimb ischemia (HLI) model.
\end{abstract}

Methods: Murine wound healing model-24-month-old aged mice received topical application of rHDL (50 $\mathrm{\mu g} / \mathrm{wound} /$ day) or PBS (vehicle control) for 10 days following wounding. Murine HLI model-Femoral artery ligation was performed on 24-month-old mice. Mice received rHDL (40 mg/kg) or PBS, intravenously, on alternate days, 1 week pre-surgery and up to 21 days post ligation. For both models, blood flow perfusion was determined using laser Doppler perfusion imaging. Mice were sacrificed at 10 (wound healing) or 21 (HLI) days post-surgery and tissues were collected for histological and gene analyses.

Results: Daily topical application of $\mathrm{rHDL}$ increased the rate of wound closure by Day 7 post-wounding $(25 \%, p<0.05)$. Wound blood perfusion, a marker of angiogenesis, was elevated in rHDL treated wounds (Days 4-10 by 22-25\%, $p<0$. 05). In addition, rHDL increased wound capillary density by $52.6 \%$. In the HLI model, rHDL infusions augmented blood flow recovery in ischemic limbs (Day 18 by $50 \%$ and Day 21 by $88 \%, p<0.05$ ) and prevented tissue necrosis and toe loss. Assessment of capillary density in ischemic hindlimb sections found a $90 \%$ increase in rHDL infused animals. In vitro studies in fibroblasts isolated from aged mice found that incubation with rHDL was able to significantly increase the key pro-angiogenic mediator vascular endothelial growth factor (VEGF) protein $(25 \%, p<0.05)$.

Conclusion: $\mathrm{rHDL}$ can promote wound healing and wound angiogenesis, and blood flow recovery in response to ischemia in aged mice. Mechanistically, this is likely to be via an increase in VEGF. This highlights a potential role for HDL in the therapeutic modulation of age-impaired vascular complications.

Keywords: Aging, Vascular complications, High-density lipoproteins, Angiogenesis, Wound repair

Abbreviations: apo A-I, Apolipoprotein A-l; HDL, High density lipoproteins; HIF-1a, Hypoxia inducible factor 1-a; HLI, Hindlimb ischemia (model); PBS, Phosphate buffered saline; PHD, Proyl hydroxylase; rHDL, Reconstituted HDL; VEGF, Vascular endothelial growth factor

\footnotetext{
* Correspondence: bursillc@hri.org.au

${ }^{1}$ The Heart Research Institute, 7 Eliza Street, Newtown, Sydney 2042, Australia

Sydney Medical School, University of Sydney, Camperdown 2050, Sydney,

Australia

Full list of author information is available at the end of the article
} 


\section{Background}

Angiogenesis is an essential element in the regenerative response to tissue damage. It occurs in healing wounds and in response to ischemic injury. However, in advanced age, this process becomes impaired and is characterized by endothelial cell decline, altered inflammatory response and impaired neovascularization [1-3]. Consequently, impaired wound healing and disordered ischemia-mediated angiogenesis become defining features of age-impaired vascular complications. Aged individuals have higher mortality and increased rates of limb amputation as a result of acute limb ischemia [4]. In these circumstances, aged patients and animal models are reported to exhibit reduced tissue production of vascular endothelial growth factor (VEGF) and decreased hypoxia inducible factor (HIF)- $1 \alpha$ stability and activation [5, 6]. Whilst this provides functional and mechanistic evidence for age-impaired wound healing and ischemia-mediated angiogenesis, the key triggers for these events have not been entirely elucidated. Recent data also indicate that processes fundamental to aging also play pivotal roles in cardiovascular disease associated with advanced age [7].

We have recently shown that high-density lipoproteins (HDL) augment ischemia driven angiogenesis in vivo and in vitro [8]. These effects in vitro were mediated via a stabilization of HIF-1 $\alpha$ [9] and an elevation in VEGF [8]. We have also recently identified that reconstituted HDL (rHDL) rescues diabetes-impaired neovascularization [10]. Given the distinct mechanistic similarities between the impairment of angiogenesis in diabetes and in aging, we now seek to determine if rHDL can also improve age-impaired angiogenesis. We investigated this using two surgical murine models of wound healing and hindlimb ischemia and find that rHDL augments wound closure and hindlimb angiogenesis in aged mice. Clinical trials that have raised HDL in patients with advanced atheroma have been disappointing showing no benefit on advanced plaque size, which may be related to the age-induced failure of HDL function in the context of advanced atherosclerosis and aging. Our studies highlight an alternate therapeutic use of HDL for angiogenesis-related diseases that is effective in the context of aging, which may facilitate its eventual successful translation in clinic.

\section{Methods}

\section{Preparation of discoidal reconstituted HDL}

Apolipoprotein A-I (apoA-I) was isolated from plasma obtained from healthy humans by ultracentrifugation and anion-exchange chromatography, as described previously [11]. The collection of human blood complied with the ethical rules for human experimentation as stated in the 1975 Declaration of Helsinki. Ethics were obtained from the Sydney Local Health District Ethics Review Committee. Discoidal reconstituted HDL (rHDL) was prepared by cholate dialysis, complexing 1palmitoyl-2-linoleoyl-phosphatidylcholine (PLPC) and apoA-I at an initial PLPC:apoA-I molar ratio of 100:1 (final PLPC:apoA-I molar ratio of 80:1). Protein concentration of rHDL was determined using bicinchoninic acid (BCA) assay (Thermo Scientific).

\section{Murine wound healing model}

All experimental procedures and protocols were conducted with the approval from the Sydney Local Health District Animal Welfare Committee, and conformed to the Guide for the Care and Use of Laboratory Animals (United States National Institute of Health). Aged male 24-month-old C57Bl/6 J mice were used for both studies. The wound healing surgery was conducted as previously described [12]. Two full-thickness excisions that include the panniculus carnosus were created on the dorsum, one on each side of the midline of the mouse. A $0.5 \mathrm{~mm}$ thick silicone splint (Life Technologies, CA, USA) was then placed around the wound with adhesive and interrupted sutures. For each mouse, one wound received $\mathrm{rHDL}(50 \mu \mathrm{g} /$ wound/day) and the other PBS (vehicle control), topically applied daily. A translucent occlusive dressing (Opsite ${ }^{\mathrm{TM}}$ Flexfix $^{\mathrm{TM}}$, Smith \& Nephew, London, UK) was then applied. Digital images were taken and micro-callipers were used to measure the wound area daily. Blood perfusion in the wound areas was determined using laser Doppler perfusion imaging (moorLDI2-IR, Moor Instruments, Devon, UK). Ten days after wounding, both wounds were excised for histological and gene analyses.

\section{Hindlimb ischemia model}

The hindlimb ischemia model was conducted as described previously [8]. The left femoral artery and vein were ligated (7-0 silk) above both the epigastrica and profunda femoris before severing them distal to the ligation. The femoral artery and vein were also completely excised as distal as the popliteal from the hindlimb of mice. A sham procedure was performed on the opposite hindlimb. Mice received i.v. injections of PBS (vehicle control) or $\mathrm{rHDL}(40 \mathrm{mg} / \mathrm{kg}$ ) every second day following surgery. Hindlimb blood reperfusion was determined by laser Doppler perfusion imaging (moorLDI2-IR, Moor Instruments) and performed prior to and immediately following surgery, then at Days 2, 4, 7, 10, 13, 16, 18 and 21 post-surgery. Following sacrifice, the gastrocnemius muscles of both ischemic and nonischemic limbs were collected for histological and gene expression analyses. 


\section{Immunohistochemistry}

Wound tissues were fixed in paraformaldehyde (4\%) and embedded in paraffin. Wound sections $(5 \mu \mathrm{m})$ were taken from the mid-point and assessed for the presence of $\mathrm{Mac}_{-} 3^{+}$macrophages (1:100, Santa Cruz) and CD31 ${ }^{+}$ neovessels (1:100, Abcam) [10]. Gastrocnemius muscle sections were frozen in OCT and fixed in $100 \%$ methanol. Hindlimb sections $(5 \mu \mathrm{m})$ were assessed for the presence of $\mathrm{CD}^{+} 1^{+}$neovessels (1:1000, Abcam), $\alpha-$ smooth muscle actin $^{+}$arterioles (1:500, Sigma Aldrich) and laminin (1:300, Merck Millipore) [10]. All images were analyzed using Image-Pro Plus 4.5 software (Diagnostic Instruments, USA). The percentage of positive staining was calculated for each high power field (minimum of 3 fields per section) and the mean staining area for each section calculated.

\section{Histochemistry}

To determine collagen deposition, paraffin embedded wound sections were stained with Milligan's trichrome stain. All images were analyzed using Image-Pro Plus 4.5 software (Diagnostic Instruments, USA). The percentage of positive staining was calculated for each high power field (minimum of 3 fields per section) and the mean staining area for each section calculated.

\section{qRT-PCR}

Total RNA was isolated from wounds and hindlimbs with TRI reagent (Sigma Aldrich). The amount of RNA was quantitated spectrophotometrically using a NanoDrop $^{\text {TM }}$ (Thermo Scientific). 200 ng total RNA was reverse transcribed using the iScript cDNA synthesis kit (Bio-Rad) in triplicate. All amplicons were amplified using iQ SYBR Green Supermix (Bio-Rad) in a Bio-Rad Cfx384 thermocycler and 20 pmol each of forward and reverse primer for murine VEGF (F: 5'-GGCTGCTGT AACGATGAAG-3'; R: 5'-CTCTCTATGTGCTGGCTT TG-3'), HIF-1 $\alpha$ (F: 5'-TCCCTTGCTCTTTGTGGTTG GGT-3'; R: 5'-AACGTAAGCGCTGACCCAGG-3'), Siah1 (F: 5'-GACTGCTACAGCATTACCCACT-3'; R: 5'-GTTG GATGCAGTTGTGCCG-3'), Siah2 (F: 5'-CTAACGCCC AGCATCAGGAA-3'; R: 5'-GAACAGCCCGTGGTAGCA TA-3'), PHD2 (F: 5'-ATCACCTGGATCGAGGGCAA- 3'; R: 5'-CGTTCGGCCGTTTATCCTGT-3'), PHD3 (F: 5'-G AGCCGGCTGGGCAAATACT-3'; R: 5'-GGGGTTGTCC ACATGGCGAA -3') and $\beta 2$-microglobulin (B2M) measured as a reference gene (F: 5'-CAACGGCAGCATTTAT AACCC-3'; R: 5'-CCCATTGATGATGGAGTGTGG-3'). Relative changes in mRNA levels of the genes of interest were normalized using the ${ }^{{ }^{\Delta}} \mathrm{CT}$ method to murine B2M.

\section{ELISA}

Wound and hindlimb tissue samples were homogenized with lysis buffer $(80 \mathrm{mM}$ Tris $\mathrm{HCl}, 10 \mathrm{mM} \mathrm{NaCl}, 50 \mathrm{mM}$
$\mathrm{NaF}, 5 \mathrm{mM} \mathrm{Na} \mathrm{P}_{2} \mathrm{O}_{7}, 15 \mathrm{nM}$ Triton-X). Protein levels of mouse VEGF were determined from wound and hindlimb lysates using commercially available human VEGF ELISA kit (R\&D Systems Inc).

\section{Plasma lipid concentration}

Total cholesterol concentrations were determined enzymatically on mouse plasma using commercially available kits (Roche Diagnostics). HDL cholesterol concentrations were determined by enzymatic assay following precipitation of apolipoprotein B containing lipoproteins with polyethylene glycol. LDL levels were determined by subtracting total HDL from total cholesterol [8]. Triglyceride levels were determined enzymatically on mouse plasma using commercially available kits (Roche Diagnostics).

\section{In vitro studies}

Fibroblasts isolated from aged mice (24 months) were grown to $80 \%$ confluency in DMEM media in hypoxia $\left(1 \% \mathrm{O}_{2} / 5 \% \mathrm{CO}_{2}\right)$ and then incubated with either PBS (control) or $\mathrm{rHDL}(600 \mu \mathrm{g} / \mathrm{ml})$ for $16 \mathrm{~h}$ [8]. Treated cells were lysed with radioimmunoprecipitation assay (RIPA) lysis buffer $(20 \mathrm{mM}$ Tris- $\mathrm{HCl}, 1 \mathrm{mM}$ EDTA, $1 \mathrm{mM}$ EGTA, $1 \mathrm{mM}$ dithiothreitol, $0.5 \mathrm{mM}$ phenylmethylsulfonyl fluoride, $1.5 \mu \mathrm{g} / \mathrm{mL}$ aprotinin, $1 \mu \mathrm{g} / \mathrm{mL}$ leupeptin, $1 \mu \mathrm{g} / \mathrm{mL}$ pepstatin, $1 \mathrm{mM}$ sodium orthovanadate, and $0.2 \%$ Triton X-100; $\mathrm{pH} 7.4$ ). The protein concentration was determined using the BCA Protein Assay (Thermo Scientific, Waltham, MA, USA). Nitrocellulose membranes were incubated in a primary antibody against VEGF (1:1000, ab46154, Abcam, Cambridge, UK). Even protein loading was confirmed with $\alpha$-tubulin (1:1000, ab40742, Abcam). Three donors were tested, each in quintuplicate ( $n=15$ total).

\section{Statistical analysis}

Data are expressed as mean \pm SEM. Differences between treatment groups were calculated using unpaired two tailed t-test or one-way ANOVA with Bonferroni's comparison test post hoc. Significance was set at a two-sided $p<0.05$.

\section{Results \\ Topical rHDL promotes wound closure and wound} angiogenesis in aged mice

We investigated the effect of topically applied rHDL in a murine model that mimics human wound repair in aged mice. Topical treatment of wounds with rHDL $(50 \mu \mathrm{g} /$ wound/day), in 24-month-old mice, showed significantly faster wound closure at Day $7(25.6 \%, p<0.01)$ and Day $8(22.2 \%, p<0.05)$ compared to PBS treated wounds (Fig. 1a). Consistent with this, blood perfusion determined by laser Doppler imaging, as a marker of wound angiogenesis, was significantly elevated in rHDL treated 


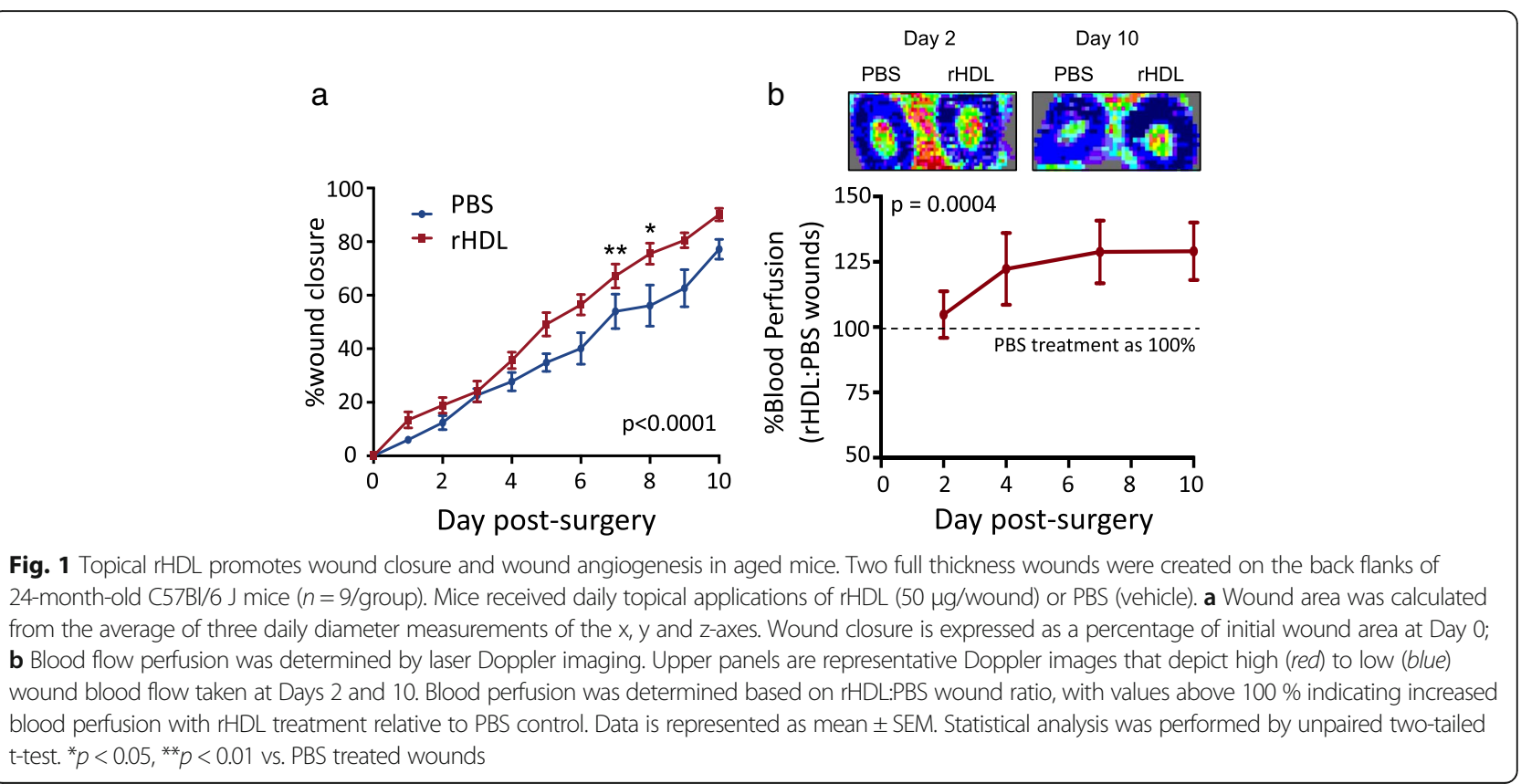

wounds from Days 4-10 (22-25 \%, $P<0.05)$, compared to PBS control (Fig. 1b).

\section{The effect of rHDL on wound neovascularization, wound macrophage content and collagen deposition}

Wound sections were stained for CD31, a marker of neovessels. Despite a $52.6 \%$ increase in $\mathrm{CD} 1^{+}$stained neovessels in rHDL treated wounds, there was no significant difference in the number of neovessels between wounds administered PBS or rHDL (Fig. 2a). Assessment of macrophage infiltration in wound sections with Mac3 showed a non-significant increase in rHDL treated wounds (Fig. 2b). Finally, we found that there was no difference in collagen deposition between rHDL and PBS treated wounds (Fig. 2c).

\section{rHDL had no effect on gene expression of angiogenic markers in wounds}

To elucidate the mechanism for the effects of rHDL on wound angiogenesis in aged mice, we examined the effect of rHDL on the key angiogenic growth factor vascular endothelial growth factor (VEGF). We found that topical application of rHDL had no impact on wound VEGF mRNA levels compared to PBS control wounds (Fig. 3a). Furthermore, treatment with rHDL did not affect VEGF protein levels in wounds (Fig. 3b). Hypoxia inducible factor (HIF)- $1 \alpha$, a key transcription factor that promotes VEGF expression in hypoxia, was not changed in wounds after topical rHDL treatment at Day 10 post-wounding (Fig. 3c). Examination of the post-translational modulators of HIF$1 \alpha$ found that there were no differences in the ubiquitin ligases Siah1 and Siah2 mRNA levels between rHDL and
PBS treated wounds (Fig. 3d - e). Similarly, both proyl hydroxylases PHD2 and PHD3 showed no difference between rHDL and the PBS control group (Fig. $3 \mathrm{f}-\mathrm{g}$ ).

rHDL promotes ischemia-induced blood perfusion in aged mice

Laser Doppler perfusion imaging showed that blood flow was equally impaired across both treatment groups following ligation (Fig. 4). However, systemic rHDL infusions augmented blood flow in the ischemic hindlimbs at all time points, reaching significance at Day 18 (50\%, $p<0.05)$ and Day $21(88 \%, p<0.05)$, compared to PBS infused control mice (Fig. 4).

\section{rHDL prevents tissue necrosis in ischemic hindlimbs of} aged mice

In support of our observations of improved blood flow in rHDL infused aged mice, we observed that in ischemic hindlimbs there was no loss of toes in mice that had been infused with rHDL (Fig. 5). In contrast, in the hindlimbs of control mice that had been infused with PBS, the loss of toes occurred in $>60 \%$ of mice. The loss of toes occurred in the third week post-ligation and is consistent with the failure of the control mice to mount a substantial blood flow recovery response. The ability of rHDL to prevent tissue necrosis is likely to be due to the promotion of blood flow perfusion in the final week in these mice.

The effect of rHDL on hindlimb neovascularization Next, we sought to determine the effect of rHDL on neovascularization by assessing capillary and arteriole 

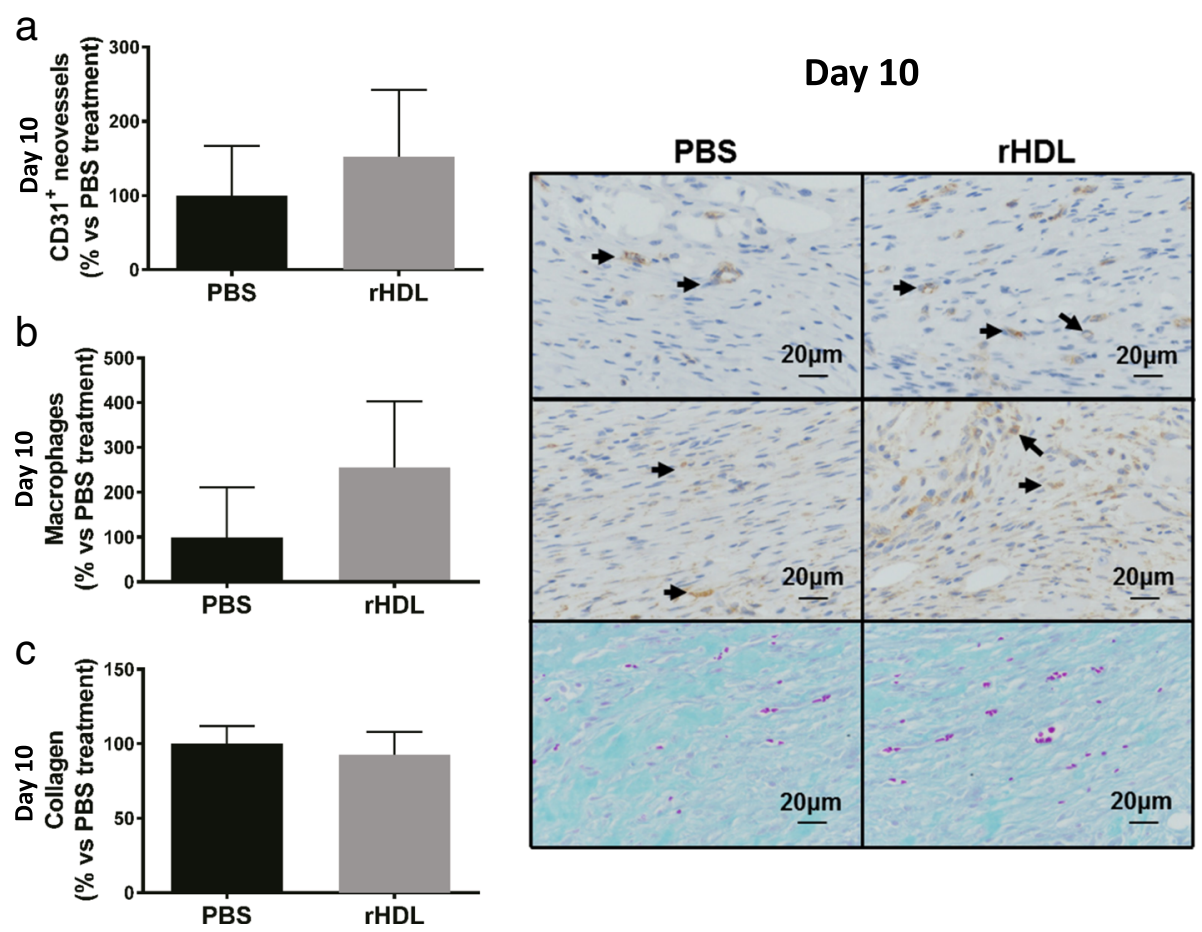

Fig. 2 The effect of $\mathrm{rHDL}$ on wound neovascularization, wound macrophage content and collagen deposition. Two full thickness wounds were created on the back flanks of 24-month-old C57BI/6 J mice ( $n=9 /$ group). Mice received daily topical applications of $\mathrm{rHDL}(50 \mathrm{\mu g} / \mathrm{wound})$ or PBS (vehicle). On Day 10 post-wounding, wound tissue was collected and sectioned for: a Capillaries using immunohistochemistry for CD31 ${ }^{+}(b r o w n$, denoted by arrows); b Macrophage infiltration was identified in wound sections using immunohistochemistry for Mac- $3^{+}$(brown, denoted by arrows); c Collagen deposition was determined using Milligan's trichrome stain (blue). Photomicrographs represent wounds stained for CD31 Mac- $3^{+}$and collagen of PBS and rHDL wounds following sacrifice. Data is represented as mean \pm SEM

density in ischemic and non-ischemic hindlimbs. Despite $>90 \%$ increases in $\mathrm{CD}_{3} 1^{+}$neovessels in both the ischemic and non-ischemic hindlimbs of rHDL infused mice, this did not reach significance when compared to control PBS infused mice (Fig. 6a). Assessment of $\alpha$ actin $^{+}$arterioles revealed that there were very few arterioles and that rHDL infusions did not change arteriole number, in either ischemic or non-ischemic hindlimbs, compared to PBS control (Fig. 6b).

\section{The effect of rHDL on angiogenic markers in ischemic hindlimbs}

Examination of the post-translational HIF- $1 \alpha$ modulators Siah1 and Siah2 (Fig. 7a - b) and PHD2 and PHD3 (Fig. 7c $-\mathrm{d}$ ) showed no significant effects on mRNA levels in both ischemic and non-ischemic hindlimbs. rHDL treated mice showed a non-significant trend toward increased HIF-1 $\alpha$ expression in ischemic hindlimbs (Fig. 7e). VEGF mRNA levels were unexpectedly lower in the ischemic hindlimbs of rHDL infused mice compared to PBS control, 21 days post-ligation $(p<0.05$; Fig. 7f). Furthermore, when hindlimb VEGF protein levels were determined, no difference was found across both groups (Fig. 7g).
rHDL increases VEGF in vitro in cells from aged mice Incubation with rHDL caused a significant increase (25\%, $p<0.0 \%$ ) in the key pro-angiogenic factor VEGF in fibroblasts isolated from aged mice (Fig. 8).

rHDL infusions increase plasma total cholesterol and HDL cholesterol

Systemic rHDL infusions elevated both total cholesterol $(p<0.01$; Fig. 9a) and HDL cholesterol ( $p<0.01$; Fig. 9b), compared to PBS infused control mice. rHDL did not affect plasma LDL or triglyceride levels (Fig. 9c - d). This increase in HDL indicates that the tail vein injections were delivered successfully.

\section{Discussion}

Wound healing is a complex dynamic process and in healthy humans consists of four distinct, but overlapping, phases including: inflammation, granulation, re-epithelialization and tissue remodelling [13]. In advanced age, however, this process becomes disrupted, and is instead characterized by an altered inflammatory response, delayed re-epithelialization and impaired angiogenesis [14]. Impaired angiogenesis is also associated with age [15] and numerous studies utilizing subcutaneous 

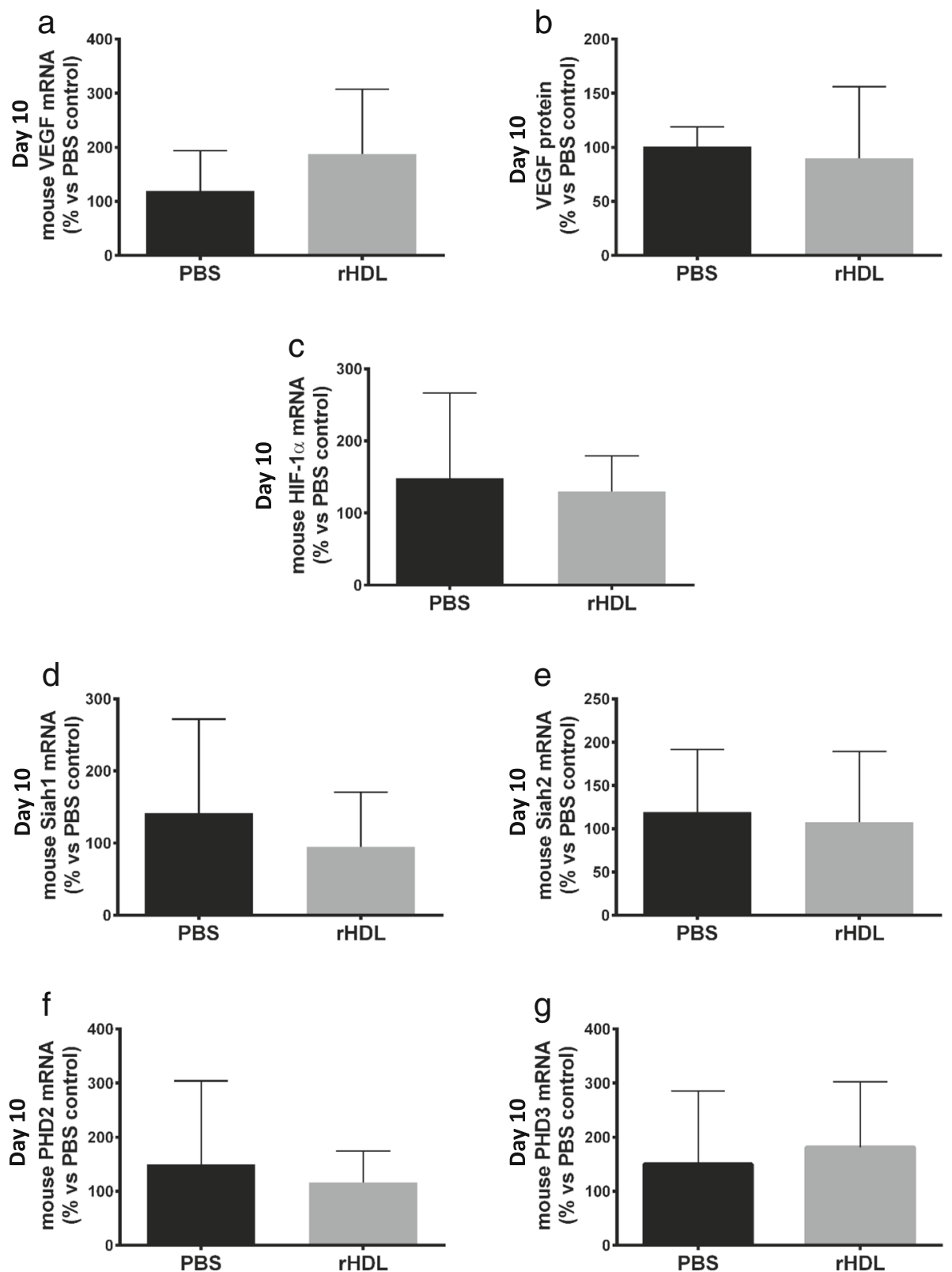

Fig. $3 \mathrm{rHDL}$ had no effect on gene expression of angiogenesis markers in wounds. Two full thickness wounds were created on the back flanks of 24-month-old C57BI/6 J mice ( $n=9 /$ group). Mice received daily topical applications of rHDL ( 50 mg/wound) or PBS (vehicle). On Day 10 post-wounding, wound tissue was collected and RNA was isolated for qRT-PCR to assess gene expression or wound tissue was homogenized and used in ELISAs to assess protein. Measured markers include: a VEGF mRNA; b VEGF protein; c HIF-1a mRNA; d Siah1 mRNA; e Siah2 mRNA; f PHD2 mRNA; and g PHD3 mRNA. Data is represented as mean \pm SEM

implant and excisional models have shown delayed wound neovascularization in aged animals $[16,17]$. The current study found that topical application of rHDL improved wound repair and wound angiogenesis in aged mice. Furthermore, systemic delivery of rHDL augmented blood flow recovery and prevented tissue necrosis in response to ischemia. Mechanistically these changes may be mediated through an increase in VEGF.
These studies show the efficacy of HDL in the rescue of aged-impaired wound repair and angiogenesis.

The potent vasculo-protective properties of HDL are well known [18] and previous studies from our lab have shown that in young mice rHDL augment wound closure and ischemia-driven angiogenesis $[8,19]$. This is the first study to show that rHDL can still effectively improve wound closure and angiogenesis in aged mice in 


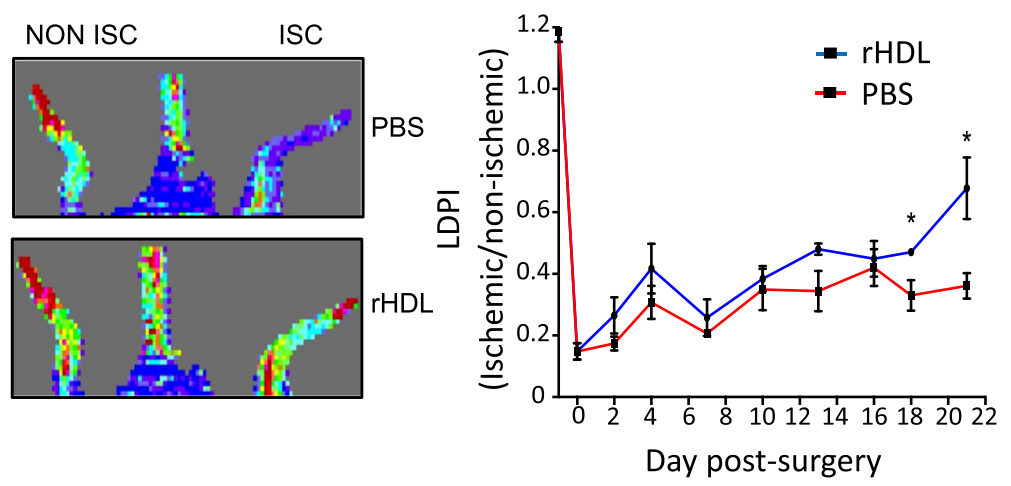

Fig. $4 \mathrm{rHDL}$ promotes ischemia-induced blood perfusion in aged mice. Femoral artery ligation was performed on 24-month-old C57B1/6 J mice $(n=6-8)$ to induce ischemia in hindlimbs. Mice received intravenous injections of PBS or $\mathrm{rHDL}(40 \mathrm{mg} / \mathrm{kg})$ on alternate days following ligation until sacrifice 21 days later. Images represent high (red) or low (blue) blood flow in animals treated with PBS or rHDL at Day 21. Laser Doppler Perfusion Index (LDPI) was determined as a ratio of ischemic (ISC) to non-ischemic (NON ISC) hindlimbs. Data is represented as mean \pm SEM. Statistical analysis was performed by unpaired two-tailed t-test. ${ }^{*} p<0.05$ vs. PBS control

which there are impairments in normal healing responses. Recent (and current) clinical trials that have raised HDL have all been aimed at reducing advanced atherosclerotic plaques in elderly individuals [20-23]. These studies have been disappointing, as they have shown no cardiovascular benefit despite increases in HDL of $11-70 \%$. This puts into question HDL raising as a therapy to reduce advanced plaque. A number of mechanisms may contribute to this failure including the reduced ability of HDL to efflux cholesterol from advanced necrotic plaques in aged individuals [24]. Furthermore, studies have found that the anti-atherogenic and anti-oxidant properties of HDL become compromised with aging and in patients with advanced coronary artery disease (CAD). HDL isolated from elderly individuals induces less macrophage cholesterol efflux and is less able to inhibit LDL oxidation when compared to HDL from young individuals [24]. HDL isolated from elderly individuals is more susceptible to oxidation than HDL isolated from young individuals [25], which has been attributed to age-related decreases in PON1 [26].
HDL from CAD patients fails to activate eNOS or NO. This has also been attributed to reductions in HDLassociated PON1 [26]. Finally, oxidatively modified apoA-I has impaired cholesterol efflux, is pro-inflammatory and is abundant in established plaques [27, 28]. Collectively, these studies suggest HDL functionality is compromised when raised in an aged/diseased milieu in the context of CAD and perhaps dietary antioxidant intake could counteract this, although the data are still conflicting as to their effectiveness [29]. Our studies, however, clearly show that if targeted to the right pathology such as wound repair and impaired ischemia-mediated angiogenesis, rHDL can still be strikingly effective in the context of aging. These findings may be important for the targeting of the HDL raising interventions in future clinical studies.

To further examine the impact of topical rHDL treatment, wounds were assessed for markers of angiogenesis and wound repair. The initial inflammatory phase of wound healing is distinguished by the infiltration of macrophages, which release pro-inflammatory growth factors such as VEGF, to promote vascular permeability and

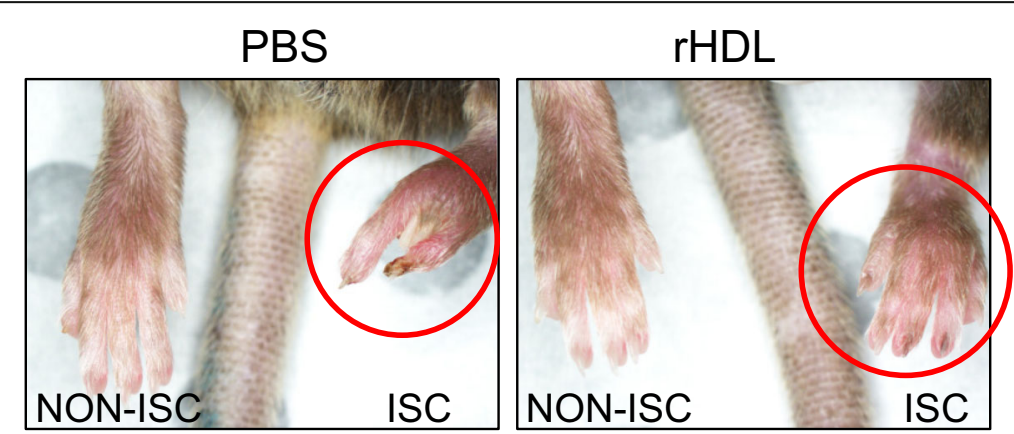

Fig. $5 \mathrm{rHDL}$ prevents tissue necrosis in ischemic hindlimbs of aged mice. Femoral artery ligation was performed on 24-month-old C57B1/6 J mice $(n=6-8)$ to induce ischemia in hindlimbs. Mice received intravenous injections of PBS or $\mathrm{rHDL}(40 \mathrm{mg} / \mathrm{kg})$ on alternate days following ligation until sacrifice 21 days later. Images are representative mouse feet at Day 21 


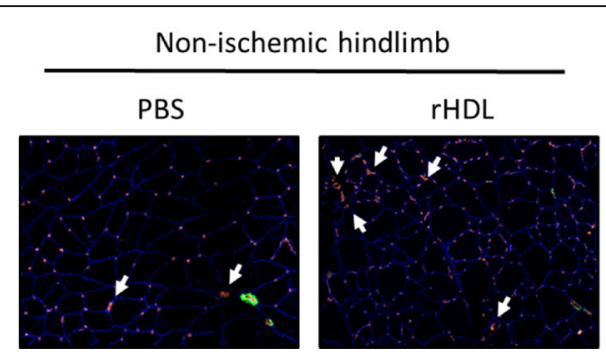

a

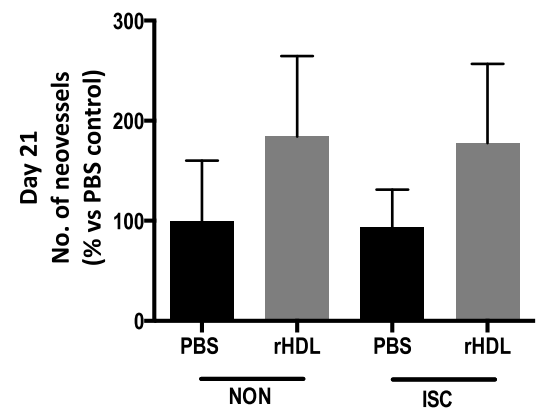

Ischemic hindlimb

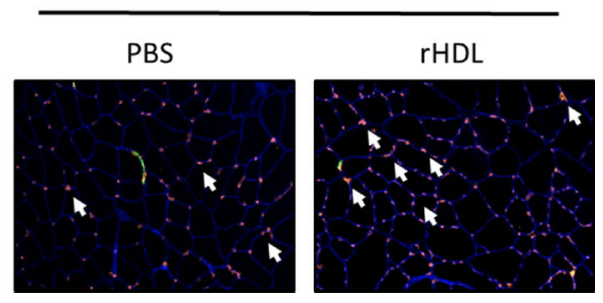

b

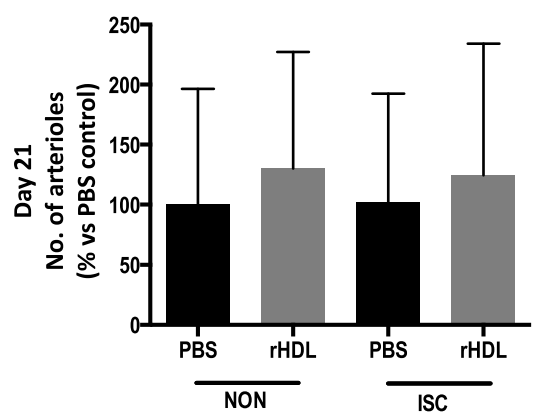

Fig. 6 The effect of $\mathrm{rHDL}$ infusions on hindlimb neovascularization. Femoral artery ligation was performed on 24-month-old C57B1/6 J mice. Mice received i.v. injections of PBS or $\mathrm{rHDL}(40 \mathrm{mg} / \mathrm{kg}$ ) on alternate days following ligation until sacrifice 21 days later. Upper panels are representative images of stained hindlimb sections at the 21-day time point. White arrows highlight capillaries. a Capillaries were identified in non-ischemic (NON) and ischemic (ISC) hindlimb sections using immunocytochemistry for CD31 ${ }^{+}$(red staining). (b) Arterioles were identified in non-ischemic and ischemic hindlimb sections using immunocytochemistry for a-actin ${ }^{+}$(green staining). Data is represented as mean \pm SEM

fibroblast activity. Age related alterations in wound repair are marked by delayed macrophage infiltration in young and middle-aged mice [30]. The next phase of wound healing is characterized by angiogenesis (capillary growth), granulation and ultimately collagen synthesis [31]. We found that there were non-significant increases in wound capillaries and macrophages. The lack of significant effect is likely because the increase in the inflammatory response and requirement for angiogenesis post-wounding occurs in the early stages of wound repair. Our histological analysis was performed on wound sections from mice at sacrifice (Day 10), a time point at which the importance of these repair processes are declining. Another contributing factor to the lack of significant increase in wound macrophage content is that HDL has potent anti-inflammatory properties and has been shown to reduce macrophage infiltration in atherosclerotic plaques [32]. This anti-inflammatory effect of HDL may therefore have counteracted any increases in wound macrophage content as part of the repair process. We also found no changes in wound collagen content between PBS and rHDL treated wounds. Collagen composition in old mice does not differ to that in young mice, however, in mature wounds there is decreased collagen deposition associated with age [15]. Our studies suggest that rHDL has no effect on the ability of a wound to deposit collagen.

VEGF is a key contributor to multiple components of the wound healing cascade including angiogenesis, epithelialization and collagen deposition [33]. Reduced levels of VEGF are associated with delayed capillary growth [15] and VEGF replacement can reverse impaired angiogenesis in aged mice [34]. Hypoxia-inducible factor$1 \alpha(\mathrm{HIF}-1 \alpha)$ is induced in ischemic conditions to promote angiogenesis and in turn promote the expression of potent angiogenic factors such as VEGF. Furthermore, HIF$1 \alpha$ is also post-translationally modulated. Upon hypoxic stimulation, the E3 ubiquitin ligases Siah1 and Siah2 are induced which suppress the expression of prolyl hydroxylases (PHDs) PHD2 and PHD3. Under normoxic conditions, the PHDs induce the ubiquitination and degradation of HIF- $1 \alpha$ by hydroxylating specific prolyl residues. Studies from our laboratory have shown that HDL augments ischemia driven angiogenesis in vivo and in vitro [8] and that these effects are mediated via the post-translational stabilization of HIF-1 $\alpha$ [9]. Based on our previous findings with HDL and as rHDL treatment increased the rate of wound closure and wound angiogenesis in aged mice in the current study, it may have been expected that there would have been elevations in both VEGF and HIF- $1 \alpha$ in the tissues of the animals. We did not, however, find any differences in these key angiogenic factors. The lack of change may be due to the time point at which the wounds were analyzed. VEGF is induced early in the wound healing process to stimulate wound angiogenesis and promote healing. Following this initial 

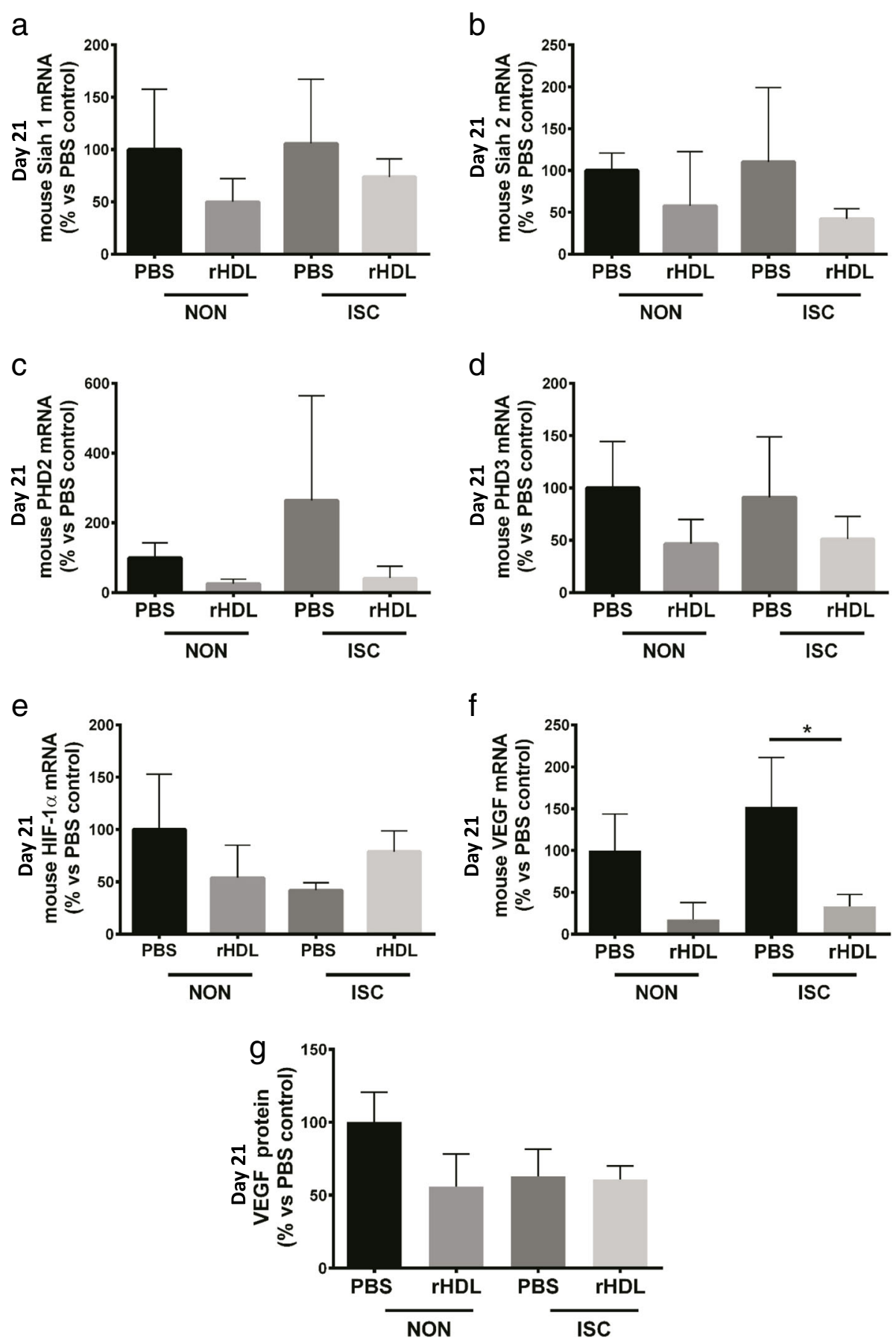

Fig. 7 The effect of $r H D L$ on angiogenic markers in ischemic hindlimbs. Femoral artery ligation was performed on 24-month-old C57B1/6 J mice. Mice received i.v. injections of PBS or rHDL $(40 \mathrm{mg} / \mathrm{kg}$ ) on alternate days following ligation until sacrifice 21 days later. On Day 21 post-surgery hindlimb tissue was collected and RNA isolated for qRT-PCR on non-ischemic (NON) and ischemic (ISC) hindlimbs to assess gene expression of (a) Siah1; (b) Siah2; (c) PHD2; (d) PHD3; (e) HIF-1a; and (f) VEGF, normalized to ß2-microglobulin (B2M). $\mathbf{g}$ VEGF protein was determined on homogenized hindlimbs using ELISA. Data is represented as mean \pm SEM. Statistical analysis was performed by one-way ANOVA and Bonferroni's comparison test post hoc. ${ }^{*} p<0.05$ vs. PBS controls

induction, VEGF levels then return to baseline. This is consistent with the lack of change observed in these angiogenic markers in our study at the Day 10 time point. It is also consistent with the increase VEGF in the aged fibroblasts following incubation with rHDL in our in vitro studies.

In addition to the deleterious impact that aging has on wound healing, studies have also shown that angiogenesis 


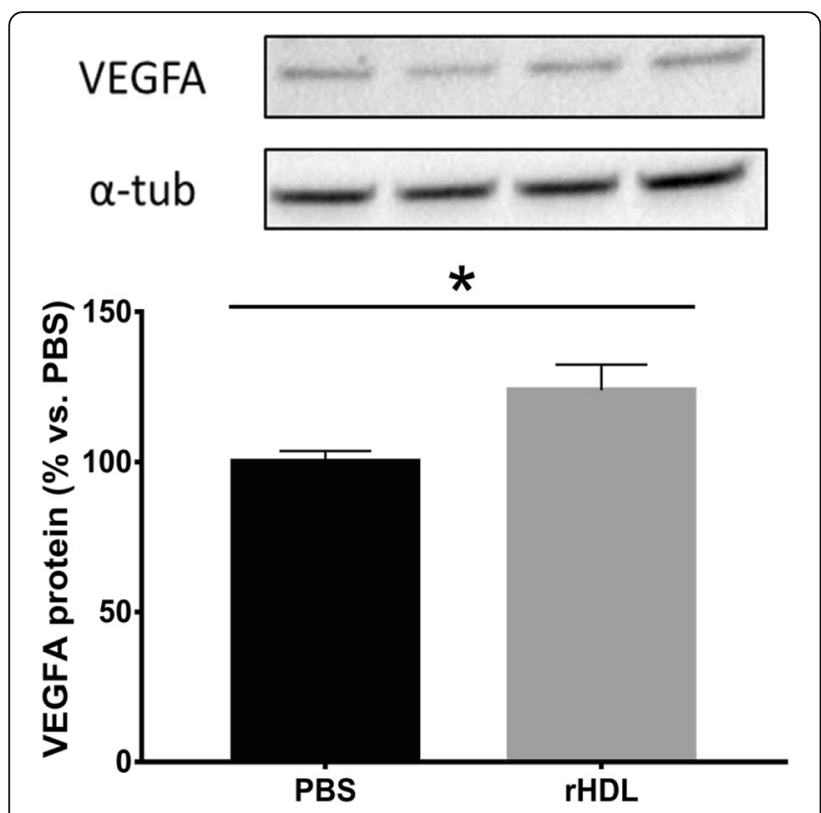

Fig. $8 \mathrm{rHDL}$ increases VEGF in fibroblasts from aged mice. Fibroblasts from aged mice were incubated with PBS (control) or rHDL for $16 \mathrm{~h}$. VEGF protein was assessed on harvested cell lysates using western blotting. Cells from three donors were tested in quintuplicate $(n=15)$. Data is represented as mean \pm SEM. ${ }^{*} p<0.05$ is responsible for collateral development (such as that seen in peripheral limb ischemia) and is impaired with aging, often leading to amputation [35]. Neovascularization is a critical part of the recovery process after ischemia [36] and aged patients show decreased capillary formation in response to ischemia [37, 38]. We have recently shown that HDL can augment ischemia-mediated angiogenesis in young mice [8]. The current study has now found that HDL can augment angiogenesis in the hindlimb ischemia model in aged animals. In the aged mice we observed that blood flow recovery was slower than in our previous work in young mice. In young mice blood perfusion starts to increase at 4-6 days post-femoral artery ligation and at 10-14 days blood perfusion levels are at $\sim 80 \%$ of the original blood flow before the ligation [8]. In the aged mice infused with PBS, blood flow recovery does not go above $40 \%$ for the entire experimental period of 21 days. Only in mice infused with rHDL does blood flow increase to $50-70 \%$ in the final experimental week. This suggests that rHDL is able to overcome age-impaired neovascularization in response to ischemia. In support of our observations of improved blood flow in rHDL infused aged mice, we observed that in ischemic hindlimbs there was no loss of toes in mice that had been infused with rHDL. The ability of rHDL to prevent tissue necrosis and toe loss is likely due to the promotion of blood flow perfusion in the final week in these aged mice. There was also a $>90 \%$ increase in hindlimb CD $31^{+}$neovessels which is consistent with the increase in blood perfusion and protection
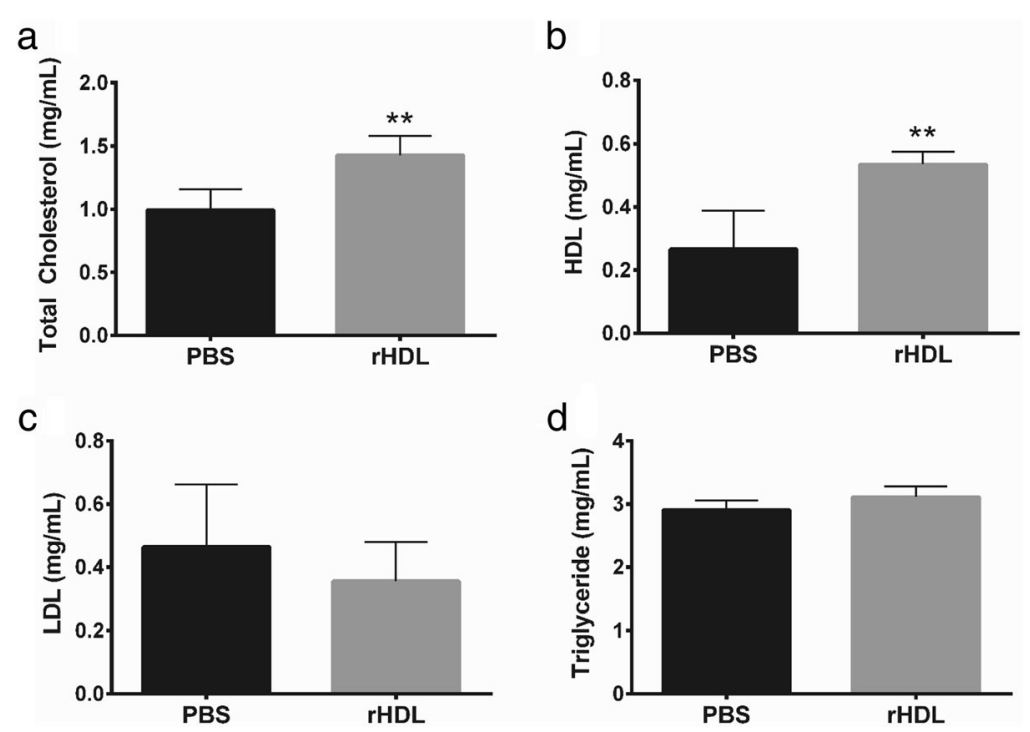

Fig. 9 rHDL infusions increase plasma total cholesterol and HDL cholesterol. Femoral artery ligation was performed on 24-month-old C57B1/6 J mice. Mice received i.v. injections of PBS or $\mathrm{rHDL}(40 \mathrm{mg} / \mathrm{kg}$ ) on alternate days following ligation until sacrifice 21 days later. Whole blood was collected via cardiac puncture at the 21 day time point and plasma isolated. a Total cholesterol concentrations were determined enzymatically on mouse plasma using commercially available kits; $\mathbf{b}$ HDL cholesterol concentrations were determined by enzymatic assay following precipitation of apolipoprotein B containing proteins with polyethylene glycol; c LDL levels were determined by subtracting total HDL from total cholesterol. $\mathbf{d}$ Triglyceride levels were determined enzymatically on mouse plasma using commercially available kits. Data is represented as mean \pm SEM. Statistical analysis was performed by unpaired two-tailed t-test. ${ }^{* *} p<0.01$ vs. PBS control 
against tissue necrosis in rHDL infused mice. We did not, however, see any changes in arterioles. Very few arterioles were detected in the hindlimb sections of these aged mice and perhaps a reflection of the delay in neovascularization and subsequent lack of maturation into arterioles by the Day 21 time point.

We have previously found that rHDL augments VEGF levels in ischemic hindlimbs in young mice [8]. This study, however, found that mRNA levels of VEGF were significantly lower in the hindlimbs of mice infused with rHDL and there was no change in hindlimb VEGF protein. The end points in our previous studies was 10 or 14 days. It is possible therefore that our results for VEGF expression in aged mice may indicate that rHDLinduced increases in hindlimb VEGF occur earlier postligation than at the 21 day time point at which they were measured.

\section{Conclusions}

In conclusion, in aged mice rHDL increased wound closure and wound angiogenesis, and augmented blood perfusion in ischemic hindlimbs, preventing tissue necrosis. These studies demonstrate the efficacy of rHDL for the treatment of angiogenesis-related diseases that are associated with aging and highlight an alternate therapeutic pathway for the translation of HDL to clinic.

\section{Funding}

This work was supported by the National Health and Medical Research Council (NHMRC) of Australia Project Grant (\#632512 to MKCN and CAB), and a Heart Foundation Career Development Fellowship (\#CR07S3331 to CAB).

\section{Availability of data materials}

Not applicable.

\section{Authors' contributions}

$C A B$ and MKCN designed the study. TT, AR, SR, LZV, YTL, JTMT and CAB performed the experiments. TT, AR, SR and JTMT analyzed the data. TT, JTMT and $C A B$ wrote the manuscript. All authors were involved in revising the final manuscript. All authors read and approved the final manuscript.

\section{Competing interests}

The authors declare that they have no competing interest.

\section{Consent for publication}

Not applicable.

\section{Author details}

${ }^{1}$ The Heart Research Institute, 7 Eliza Street, Newtown, Sydney 2042, Australia. 'Sydney Medical School, University of Sydney, Camperdown 2050, Sydney, Australia. ${ }^{3}$ Department of Cardiology, Royal Prince Alfred Hospital, Camperdown 2050, Sydney, Australia.

Received: 11 August 2016 Accepted: 31 August 2016

Published online: 06 September 2016

\section{References}

1. Zhu WH, Han J, Nicosia RF. Requisite role of p38 MAPK in mural cell recruitment during angiogenesis in the rat aorta model. J Vasc Res. 2003:40:140-8

2. Reed MJ, Edelberg JM. Impaired angiogenesis in the aged. Sci Aging Knowledge Environ. 2004;2004:pe7.
3. Reed MJ, Karres N, Eyman D, Cruz A, Brekken RA, Plymate S. The effects of aging on tumor growth and angiogenesis are tumor-cell dependent. Int J Cancer. 2007;120:753-60

4. Ouriel K, Veith FJ. Acute lower limb ischemia: determinants of outcome. Surgery. 1998;124:336-41. discussion 341-332.

5. Wagatsuma A. Effect of aging on expression of angiogenesis-related factors in mouse skeletal muscle. Exp Gerontol. 2006;41:49-54.

6. Ryan NA, Zwetsloot KA, Westerkamp LM, Hickner RC, Pofahl WE, Gavin TP. Lower skeletal muscle capillarization and VEGF expression in aged vs. young men. J Appl Physiol (1985). 2006;100:178-85.

7. Meehan B, Garnier D, Dombrovsky A, Lau K, D'Asti E, Magnus N, Rak J. Ageing-related responses to antiangiogenic effects of sunitinib in atherosclerosis-prone mice. Mech Ageing Dev. 2014;140:13-22.

8. Prosser HC, Tan JT, Dunn LL, Patel S, Vanags LZ, Bao S, Ng MK, Bursill CA. Multifunctional regulation of angiogenesis by high-density lipoproteins. Cardiovasc Res. 2014;101:145-54.

9. Tan JT, Prosser HC, Vanags LZ, Monger SA, Ng MK, Bursill CA. High-density lipoproteins augment hypoxia-induced angiogenesis via regulation of post-translational modulation of hypoxia-inducible factor 1alpha. FASEB J. 2014;28:206-17

10. Tan JT, Prosser HC, Dunn LL, Vanags LZ, Ridiandries A, Tsatralis T, Leece L, Clayton ZE, Yuen SC, Robertson S, et al. High Density Lipoproteins Rescue Diabetes-Impaired Angiogenesis via Scavenger Receptor Class B Type I. Diabetes. 2016.

11. Weisweiler P. Isolation and quantitation of apolipoproteins $A-I$ and $A-\|$ from human high-density lipoproteins by fast-protein liquid chromatography. Clin Chim Acta. 1987;169:249-54.

12. Dunn L, Prosser HC, Tan JT, Vanags LZ, Ng MK, Bursill CA. Murine model of wound healing. J Vis Exp. 2013;28(75):e50265.

13. Diegelmann RF, Kim JC, Lindblad WJ, Smith TC, Harris TM, Cohen IK. Collection of leukocytes, fibroblasts, and collagen within an implantable reservoir tube during tissue repair. J Leukoc Biol. 1987:42:667-72.

14. Gosain A, DiPietro LA. Aging and wound healing. World J Surg. 2004;28:321-6.

15. Swift ME, Kleinman HK, DiPietro LA. Impaired wound repair and delayed angiogenesis in aged mice. Lab Invest. 1999;79:1479-87.

16. Pili R, Guo Y, Chang J, Nakanishi H, Martin GR, Passaniti A. Altered angiogenesis underlying age-dependent changes in tumor growth. J Natl Cancer Inst. 1994;86:1303-14.

17. Passaniti A, Taylor RM, Pili R, Guo Y, Long PV, Haney JA, Pauly RR, Grant DS, Martin GR. A simple, quantitative method for assessing angiogenesis and antiangiogenic agents using reconstituted basement membrane, heparin, and fibroblast growth factor. Lab Invest. 1992;67:519-28.

18. Rye KA, Barter PJ. Antiinflammatory actions of HDL: a new insight. Arterioscler Thromb Vasc Biol. 2008;28:1890-1.

19. Tan JTMPH, Dunn LL, Vanags LZ, Ridiandries A, Tsatralis T, Clayton ZE, Lecce L, Robertson S, Lam YT, Ng MKC, Bursill CA. High density lipoproteins rescue diabetes-impaired angiogenesis via scavenger receptor-BI and restoration of VEGF and HIF-1a stability. Diabetes. 2015;64:A1-A100.

20. Barter PJ, Caulfield M, Eriksson M, Grundy SM, Kastelein JJ, Komajda M, Lopez-Sendon J, Mosca L, Tardif JC, Waters DD, et al. Effects of torcetrapib in patients at high risk for coronary events. N Engl J Med. 2007;357:2109-22.

21. Fayad ZA, Mani V, Woodward M, Kallend D, Abt M, Burgess T, Fuster V, Ballantyne CM, Stein EA, Tardif JC, et al. Safety and efficacy of dalcetrapib on atherosclerotic disease using novel non-invasive multimodality imaging (dal-PLAQUE): a randomised clinical trial. Lancet. 2011;378:1547-59.

22. Schwartz GG, Olsson AG, Abt M, Ballantyne CM, Barter PJ, Brumm J, Chaitman BR, Holme IM, Kallend D, Leiter LA, et al. Effects of dalcetrapib in patients with a recent acute coronary syndrome. $\mathrm{N}$ Engl Med. 2012;367:2089-99.

23. Boden WE, Probstfield JL, Anderson T, Chaitman BR, Desvignes-Nickens $P$, Koprowicz K, McBride R, Teo K, Weintraub W. Niacin in patients with low $\mathrm{HDL}$ cholesterol levels receiving intensive statin therapy. N Engl J Med. 2011;365:2255-67.

24. Berrougui H, Isabelle M, Cloutier M, Grenier G, Khalil A. Age-related impairment of HDL-mediated cholesterol efflux. J Lipid Res. 2007;48:328-36.

25. Khalil A, Jay-Gerin JP, Fulop Jr T. Age-related increased susceptibility of high-density lipoproteins (HDL) to in vitro oxidation induced by gamma-radiolysis of water. FEBS Lett. 1998;435:153-8.

26. Seres I, Paragh G, Deschene E, Fulop Jr T, Khalil A. Study of factors influencing the decreased HDL associated PON1 activity with aging. Exp Gerontol. 2004;39:59-66. 
27. Huang Y, Didonato JA, Levison BS, Schmitt D, Li L, Wu Y, Buffa J, Kim T, Gerstenecker GS, Gu X, et al. An abundant dysfunctional apolipoprotein A1 in human atheroma. Nat Med. 2014;20:193-203.

28. Didonato JA, Aulak K, Huang Y, Wagner M, Gerstenecker G, Topbas C, Gogonea V, Didonato AJ, Tang WH, Mehl RA, et al. Site-specific nitration of apolipoprotein A-l at tyrosine 166 is both abundant within human atherosclerotic plaque and dysfunctional. J Biol Chem. 2014;289(15):10276-92.

29. Ciccone MM, Cortese F, Gesualdo M, Carbonara S, Zito A, Ricci G, De Pascalis F, Scicchitano P, Riccioni G. Dietary intake of carotenoids and their antioxidant and anti-inflammatory effects in cardiovascular care. Mediators Inflamm. 2013;2013:782137.

30. Ashcroft GS, Horan MA, Ferguson MW. Aging is associated with reduced deposition of specific extracellular matrix components, an upregulation of angiogenesis, and an altered inflammatory response in a murine incisional wound healing model. J Invest Dermatol. 1997:108:430-7.

31. Sunderkotter C, Steinbrink K, Goebeler M, Bhardwaj R, Sorg C. Macrophages and angiogenesis. J Leukoc Biol. 1994;55:410-22.

32. Bursill CA, Castro ML, Beattie DT, Nakhla S, van der Vorst E, Heather AK, Barter PJ, Rye KA. High-density lipoproteins suppress chemokines and chemokine receptors in vitro and in vivo. Arterioscler Thromb Vasc Biol. 2010;30:1773-8.

33. Stojadinovic O, Lee B, Vouthounis C, Vukelic S, Pastar I, Blumenberg M, Brem $\mathrm{H}$, Tomic-Canic M. Novel genomic effects of glucocorticoids in epidermal keratinocytes: inhibition of apoptosis, interferon-gamma pathway, and wound healing along with promotion of terminal differentiation. J Biol Chem. 2007:282:4021-34.

34. Arthur WT, Vernon RB, Sage EH, Reed MJ. Growth factors reverse the impaired sprouting of microvessels from aged mice. Microvasc Res. 1998:55:260-70.

35. Rivard A, Isner JM. Angiogenesis and vasculogenesis in treatment of cardiovascular disease. Mol Med. 1998;4:429-40.

36. Carmeliet P, Jain RK. Molecular mechanisms and clinical applications of angiogenesis. Nature. 2011;473:298-307.

37. Coggan AR, Spina RJ, King DS, Rogers MA, Brown M, Nemeth PM, Holloszy JO. Skeletal muscle adaptations to endurance training in 60- to 70-yr-old men and women. J Appl Physiol (1985). 1992;72:1780-6.

38. Gavin TP, Ruster RS, Carrithers JA, Zwetsloot KA, Kraus RM, Evans CA, Knapp DJ, Drew JL, McCartney JS, Garry JP, Hickner RC. No difference in the skeletal muscle angiogenic response to aerobic exercise training between young and aged men. J Physiol. 2007;585:231-9.

\section{Submit your next manuscript to BioMed Central and we will help you at every step:}

- We accept pre-submission inquiries

- Our selector tool helps you to find the most relevant journal

- We provide round the clock customer support

- Convenient online submission

- Thorough peer review

- Inclusion in PubMed and all major indexing services

- Maximum visibility for your research

Submit your manuscript at wuw biomedcentral.com/submit

) Biomed Central 